\title{
Superfield generating equation of field-antifield formalism as a hyper-gauge theory
}

\author{
Igor A. Batalin ${ }^{1,2, a}$, Peter M. Lavrov ${ }^{2,3, b}$ \\ ${ }^{1}$ P.N. Lebedev Physics Institute, Leninsky Prospect 53, 119991 Moscow, Russia \\ 2 Tomsk State Pedagogical University, Kievskaya St. 60, 634061 Tomsk, Russia \\ ${ }^{3}$ National Research Tomsk State University, Lenin Av. 36, 634050 Tomsk, Russia
}

Received: 3 February 2017 / Accepted: 13 February 2017 / Published online: 22 February 2017

(C) The Author(s) 2017. This article is published with open access at Springerlink.com

\begin{abstract}
Within a superfield approach, we formulate a simple quantum generating equation of the field-antifield formalism. Then we derive the Schroedinger equation with the Hamiltonian whose $\Delta$-exact part serves as a generator to the quantum master transformations. We show that these generators do satisfy a nice composition law in terms of the quantum antibrackets. We also present an $S p(2)$ symmetric extension to the main construction, with specific features caused by the principal fact that all basic equations become $S p(2)$ vector-valued ones.
\end{abstract}

\section{Introduction}

From the early days of the field-antifield formalism, a fundamental idea was presented [1,2] as to how to formulate a universal hyper-gauge theory whose gauge generators would, by construction, be included naturally into the Hessian of the original master action of the universal theory, defined so as to satisfy the (classical) master equation formulated in terms of the antibrackests $[3,4]$. Then the notion of a proper solution to the master equation was defined by requiring that there were no other gauge generators involved than the ones included into the Hessian. The next step was made by formulating the quantum master equations in terms of the odd Laplacian operator. The quantum master equation was derived later directly from the Hamiltonian formalism [5,6]. These basic ideas were developed as a success [1,2], as applied to both the irreducible and to the reducible gauge theories. In general, the universal hyper-gauge theory was invented so as to "be ready" to include into itself any possible particular model with a gauge-invariant initial action.

\footnotetext{
a e-mail: batalin@1pi.ru

be-mail: lavrov@tspu.edu.ru
}

In the present paper, we develop further the profound idea of the hyper-gauge theory at the quantum superfield level. Firstly, within a superfield approach [7-9], we formulate a simple quantum generating equation of the field-antifield formalism as having its configuration space identified with the antisymplectic phase space of fields and antifields. The latter generating equation is presented in terms of a superfield covariant derivative with respect to the two-dimensional super-time whose boson component is the "ordinary" time, purely formal in its origin, while its fermion component is identified naturally with the BRST parameter. The covariant derivative squared is just the "ordinary" time derivative. Then we derive the standard Schroedinger equation by applying again the covariant derivative to the generating superfield equation. We provide effectively for the Hamiltonian commuting with the odd Laplacian (the $\Delta$ operator). As usual, the Hamiltonian consists of a singlet part and a $\Delta$-exact part. In particular, in the absence of a singlet component, the Hamiltonian becomes purely $\Delta$-exact. We show that the $\Delta$-exact part of the Hamiltonian serves as a generator to the quantum master transformations. Classically, these transformations consist of two pieces: the first of them is just an anticanonical transformation, while the second is caused by the Jacobian of the transformation. Then we show that the generators of the quantum master transformations do satisfy a very nice composition law as formulated in terms of the so-called quantum antibrackets $[10,11]$. We also present an $S p$ (2) symmetric extension to the main construction, with specific features caused by the principal fact that all basic equations become $S p(2)$ vector-valued ones.

\section{Superfield generating equation}

It appears to be a remarkable feature that the generating equation of the field-antifield formalism takes the very simple 
form of a superfield Schroedinger equation,

$(i \hbar D-Q) \Psi=0, \quad D=: \frac{\partial}{\partial \tau}+\tau \frac{\partial}{\partial t}, \quad Q=: \Delta-F$,

where $D$ is a covariant super-time derivative, $Q$ is a supercharge whose kinetic part is the odd Laplacian, $\Delta$, and $F$ is a super-potential depending on the momenta in general,

$\varepsilon(D)=1, \quad D^{2}=\frac{1}{2}[D, D]=\frac{\partial}{\partial t}$,

$\varepsilon(\Delta)=1, \quad \Delta^{2}=\frac{1}{2}[\Delta, \Delta]=0$,

$F=: F(Z, P), \quad \varepsilon(F)=1$.

Equation (2.1) is formulated for a superfield,

$\Psi=: \Psi(t, \tau, Z), \quad \varepsilon(t)=0, \quad \varepsilon(\tau)=1$.

We assume that the co-ordinate operators $Z^{A}$ are identified with the standard full set of the field-antifield variables, and $P_{A}$ are their respective canonically conjugate momenta operators,

$\left[Z^{A}, Z^{B}\right]=0, \quad\left[Z^{A}, P_{B}\right]=i \hbar \delta_{B}^{A}, \quad\left[P_{A}, P_{B}\right]=0$.

It follows from (2.1) that the standard Schroedinger equation holds:

$i \hbar \frac{\partial}{\partial t} \Psi=\mathcal{H} \Psi$,

with the Hamiltonian

$\mathcal{H}=:-(i \hbar)^{-1} \frac{1}{2}[Q, Q]=(i \hbar)^{-1}\left[\left(\Delta-\frac{1}{2} F\right), F\right]$.

The superfield (2.5) has the component form

$\Psi(t, \tau, Z)=\left(1+\tau(i \hbar)^{-1} Q\right) \Psi_{0}(t, Z)$,

where the zero-component $\Psi_{0}(t, Z)$ satisfies by itself Eq. (2.7) with the Hamiltonian (2.8). As for an arbitrary $F$, the Hamiltonian (2.8) does not commute with the $\Delta$. However, it follows from (2.8) that

$[Q, \mathcal{H}]=0$.

Thus, we arrive at the implication

$[\Delta, \mathcal{H}]=0 \Rightarrow[\mathcal{H}, F]=0$

or more explicitly

$[[\Delta, F], F]=\left[\Delta, \frac{1}{2}[F, F]\right]=0$.

Due to the Poincaré lemma, we have

$\frac{1}{2}[F, F]=-i \hbar \mathcal{H}_{S}-[\Delta, G]$,

where $\mathcal{H}_{S}$ is a boson singlet component,

$\left[\Delta, \mathcal{H}_{S}\right]=0, \quad \mathcal{H}_{S} \neq[\Delta$, anything $]$,
$G$ is an arbitrary fermion operator. By inserting (2.13) into (2.8), we get

$\mathcal{H}=\mathcal{H}_{S}+\mathcal{H}_{\Phi}$,

where the $\Delta$-exact $\Phi$ component is defined as

$\mathcal{H}_{\Phi}=:(i \hbar)^{-1}[\Delta, \Phi], \quad \Phi=: F+G$.

As the $G$ in the second equation in (2.16) is an arbitrary fermion operator, the respective natural arbitrariness is inherited in (2.15), as well, with having the implicit $G$ dependence taken into account in the $F$, via Eq. (2.13) with the singlet component $\mathcal{H}_{S}$ being kept fixed. In its turn, Eq. (2.13) can be rewritten in the equivalent form

$\frac{1}{2}(i \hbar)^{-1}([G, G]-[\Phi, \Phi])=\mathcal{H}_{S}+(i \hbar)^{-1}[Q, G]$.

Once the $\Delta$ operator commutes with the Hamiltonian $\mathcal{H}$, it follows from Eq. (2.7) for the zero-component $\Psi_{0}$

$i \hbar \frac{\partial}{\partial t} \Delta \Psi_{0}=\mathcal{H} \Delta \Psi_{0}$,

$\left.\Delta \Psi_{0}\right|_{t=0}=\left.0 \Rightarrow \Delta \Psi_{0}\right|_{\text {any } t}=0$.

The implication (2.19) shows that the arbitrariness of a solution to the quantum master equation,

$\Delta \Psi_{0}=0, \quad \varepsilon\left(\Psi_{0}\right)=0, \quad \Psi_{0}=: \exp \left\{\frac{i}{\hbar} W\right\}$,

is measured by the evolution operator,

$\left.\left.\Psi_{0}\right|_{t=0} \rightarrow \Psi_{0}\right|_{\text {any } t}=\left.\exp \left\{-\frac{i}{\hbar} \mathcal{H} t\right\} \Psi_{0}\right|_{t=0}$.

\section{Quantum master transformations and their composition law}

Now, consider a family of operators

$\mathcal{H}_{F}=:(i \hbar)^{-1}[\Delta, F]$,

with $F(Z, P)$ being an arbitrary fermion operator. By definition, Eq. (3.1) is a generator of a quantum master transformation [12]. Notice that the operator (3.1) can be rewritten naturally in terms of both the free-acting operators $P_{A}$ and the adjoint-acting ones $P_{A}^{\prime}$,

$i \hbar \mathcal{H}_{F}=\left(\Delta^{\prime} F\right)-\operatorname{ad}^{\prime}(F)$,

where we have used the definitions

$\operatorname{ad}^{\prime}(F)=:\left(F \overleftarrow{P}_{A}^{\prime}\right) E^{A B} P_{B}(-1)^{\varepsilon_{B}}$

$\Delta=: \frac{1}{2} P_{A} E^{A B} P_{B}(-1)^{\varepsilon_{B}}, \quad E^{A B}=$ const,

$$
\Delta^{\prime}=:\left.\Delta\right|_{P \rightarrow P^{\prime}},
$$

$P_{A}=:-i \hbar \partial_{A}(-1)^{\varepsilon_{A}}$, 


$$
P_{A}^{\prime}=: \operatorname{ad}\left(P_{A}\right)=\left[P_{A}, \cdot\right], \quad \overleftarrow{P}_{A}^{\prime}=:-\left[\cdot, P_{A}\right]
$$

Due to the Jacobi identity for (super)commutators, the following relations hold for arbitrary operators $A, B$,

$\operatorname{ad}(A)=:[A, \cdot] \Rightarrow[\operatorname{ad}(A), \operatorname{ad}(B)]=\operatorname{ad}([A, B])$.

From the classical point of view, in the right-hand side in (3.2), the second term describes an anticanonical transformation with $F$ being a generator, while the first term is caused by the Jacobian of the latter transformation.

A solution to the Schroedinger equation (2.7) with the Hamiltonian (3.2) has the form of a quantum anticanonical transformation,

$\Psi=\exp \left\{-(i \hbar)^{-2} t \operatorname{ad}^{\prime}(F)\right\} \Psi_{J}$,

where the "Jacobian wave function", $\Psi_{J}$, does satisfy the equation

$$
\begin{aligned}
\partial_{t} \Psi_{J}= & \exp \left\{(i \hbar)^{-2} t \operatorname{ad}^{\prime}(F)\right\}(i \hbar)^{-2}\left(\Delta^{\prime} F\right) \\
& \times \exp \left\{-(i \hbar)^{-2} t \operatorname{ad}^{\prime}(F)\right\} \Psi_{J} .
\end{aligned}
$$

In the case of $F$ being a function of $Z$ only, Eqs. (3.7) and (3.8) do provide for the exact solution [12-16],

$$
\begin{aligned}
\Psi(Z, t)= & \exp \left\{t\left(E\left(-(i \hbar)^{-2} t \operatorname{ad}^{\prime}(F)\right)(i \hbar)^{-2}\left(\Delta^{\prime} F\right)\right)(Z)\right\} \\
& \times \exp \left\{-(i \hbar)^{-2} t \operatorname{ad}^{\prime}(F)\right\} \Psi_{0}(Z),
\end{aligned}
$$

where we have denoted

$F=: F(Z), \quad E(X)=: \frac{\exp \{X\}-1}{X}$,

and $\Psi_{0}(Z)$ is an initial wave function. Provided the first equation of (3.10) holds, the $Z P$ symbol for the whole operator (3.2) corresponds to the Weyl symbol for the second term alone in the latter operator [17].

It is a remarkable feature that the generators of the form (3.1) satisfy the following composition law:

$(i \hbar)^{-1}\left[\mathcal{H}_{F}, \mathcal{H}_{F^{\prime}}\right]=\mathcal{H}_{F \circ F^{\prime}}$,

where

$F \circ F^{\prime}=:(i \hbar)^{-2}\left(F, F^{\prime}\right)_{\Delta}$,

with $(A, B)_{\Delta}$ being the so-called quantum $\Delta$-antibracket [10, $11,18,19]$,

$$
\begin{aligned}
(A, B)_{\Delta}=: & \frac{1}{2}([A,[\Delta, B]] \\
& \left.-(A \leftrightarrow B)(-1)^{\left(\varepsilon_{A}+1\right)\left(\varepsilon_{B}+1\right)}\right) .
\end{aligned}
$$

Their main property,

$\left[\Delta,(A, B)_{\Delta}\right]=[[\Delta, A],[\Delta, B]]$, yields Eq. (3.11) immediately. The quantum 2-antibracket (3.13) does satisfy the modified Jacobi relations,

$$
\begin{aligned}
(A, & \left.(B, C)_{\Delta}\right)_{\Delta}(-1)^{\left(\varepsilon_{A}+1\right)\left(\varepsilon_{C}+1\right)}+\text { cyclic perm. }(A, B, C) \\
\quad= & \frac{1}{2}\left[(A, B, C)_{\Delta}(-1)^{\left(\varepsilon_{A}+1\right)\left(\varepsilon_{C}+1\right)}, \Delta\right],
\end{aligned}
$$

where the $(A, B, C)_{\Delta}$ is the so-called quantum 3 -antibracket, and so on $[10,11]$.

\section{$4 S p(2)$ symmetric construction}

In its $S p(2)$ symmetric version [20-23], a superfield Schroedinger equation becomes $S p(2)$ vector valued,

$\left(i \hbar D^{a}-Q^{a}\right) \Psi=0$,

where the following conventions ${ }^{1}$ hold for the required $S p(2)$ vector-valued operators:

$$
\begin{aligned}
& D^{a}=: \frac{\partial}{\partial \tau_{a}}+g^{a b} \tau_{b} \frac{\partial}{\partial t}, \quad\left[D^{a}, D^{b}\right]=2 g^{a b} \frac{\partial}{\partial t}, \\
& Q^{a}=: \Delta_{+}^{a}-F^{a}, \quad \Delta_{ \pm}^{a}=: \Delta^{a} \pm \frac{i}{\hbar} V^{a}, \\
& F^{a}=: g^{a b} \varepsilon_{b c}(i \hbar)^{-1}\left[\Delta_{+}^{c}, B\right], \\
& {\left[\Delta^{a}, \Delta^{b}\right]=0, \quad\left[\Delta_{ \pm}^{a}, \Delta_{ \pm}^{b}\right]=0,} \\
& Z^{A}=:\left(\Phi^{\alpha}, \Phi^{\alpha a} ;\right. \\
& \left.\quad \Phi_{\alpha a}^{*}, \Phi_{\alpha}^{* *}\right), \quad P_{A}=:\left(P_{\alpha}, P_{\alpha a} ; P_{*}^{\alpha a}, P_{* *}^{\alpha}\right), \\
& \Delta^{a}=: \frac{1}{2} P_{A} E^{A B a} P_{B}(-1)^{\varepsilon_{B}}, \quad E^{A B a}=\mathrm{const}, \\
& V^{a}=:-i \hbar \varepsilon^{a b} \Phi_{\alpha b}^{*} P_{* *}^{\alpha}(-1)^{\varepsilon_{\alpha}},
\end{aligned}
$$

a boson operator $B$ is restricted so as to satisfy the specific "master equation",

$\left[\Delta_{+}^{a},(B, B)_{\Delta_{+}}^{b}\right]+(a \leftrightarrow b)=0, \quad \varepsilon(B)=0$,

with

$$
\begin{aligned}
(A, B)_{\Delta_{ \pm}}^{a}=: & \frac{1}{2}\left(\left[A,\left[\Delta_{ \pm}^{a}, B\right]\right]\right. \\
& \left.-(A \leftrightarrow B)(-1)^{\left(\varepsilon_{A}+1\right)\left(\varepsilon_{B}+1\right)}\right),
\end{aligned}
$$

being the $S p(2)$ vector-valued quantum antibracket [11]. The main property of the quantum 2-antibracket holds, (4.9),

$$
\begin{aligned}
& {\left[\Delta_{ \pm}^{a},(A, B)_{\Delta_{ \pm}}^{b}\right]+(a \leftrightarrow b)} \\
& \quad=\left[\left[\Delta_{ \pm}^{a}, A\right],\left[\Delta_{ \pm}^{b}, B\right]\right]+(a \leftrightarrow b) .
\end{aligned}
$$

\footnotetext{
${ }^{1}$ For the sake of uniformity, henceforth we make use of the notation $\Phi^{\alpha a}$ for the former field variable $\pi^{\alpha a}$ [20]. Also, as for the boson metric $g^{a b}$, we assume it symmetric, constant, and invertible, so that $g_{a b}$ is its inverse.
} 
Also, the quantum 2-antibracket (4.9) does satisfy the modified Jacobi relation,

$$
\begin{aligned}
((A, & \left.(B, C)_{\Delta_{ \pm}}^{a}\right)_{\Delta_{ \pm}}^{b}(-1)^{\left(\varepsilon_{A}+1\right)\left(\varepsilon_{C}+1\right)} \\
& + \text { cyclic perm. }(A, B, C))+(a \leftrightarrow b) \\
\quad= & \frac{1}{2}\left(\left[(A, B, C)_{\Delta_{ \pm}}^{a}(-1)^{\left(\varepsilon_{A}+1\right)\left(\varepsilon_{C}+1\right)}, \Delta_{ \pm}^{b}\right]+(a \leftrightarrow b)\right),
\end{aligned}
$$

where the $(A, B, C)_{\Delta_{ \pm}}^{a}$ is the so-called quantum 3antibracket, and so on. In the $S p$ (2) case, Eqs. (4.9), (4.10), and (4.11) are natural counterparts to Eqs. (3.13), (3.14), and (3.15), respectively, in the $S p(1)$ case.

Due to the $S p(2)$ symmetric version of the Poincaré lemma, we have from (4.8)

$\frac{1}{2}(B, B)_{\Delta_{+}}^{a}=(i \hbar)^{2} X^{a}+i \hbar\left[\Delta_{+}^{a}, Y\right]$,

where $X^{a}$ is an $S p(2)$ vector-valued singlet fermion operator, $\left[\Delta_{+}^{a}, X^{b}\right]+(a \leftrightarrow b)=0, \quad X^{a} \neq\left[\Delta_{+}^{a}\right.$, anything $]$,

$Y$ is an arbitrary $S p(2)$ invariant boson operator, "anything" is an arbitrary $S p(2)$ invariant boson operator. In the $S p(2)$ case, Eqs. (4.8) and (4.12) are natural counterparts to the respective equations (2.12), (2.13) in the $S p(1)$ case.

Due to the property (4.2), it follows from the generating Eq. (4.1),

$i \hbar \frac{\partial}{\partial t} \Psi=\mathcal{H} \Psi$

where the Hamiltonian has the well-known form commuting certainly with the operators $\Delta_{+}^{a}$,

$$
\begin{aligned}
\mathcal{H} & =:-\frac{1}{4} g_{a b}(i \hbar)^{-1}\left[Q^{a}, Q^{b}\right] \\
& =\frac{1}{2}(i \hbar)^{-2}\left[\Delta_{+}^{a}, \varepsilon_{a b}\left[\Delta_{+}^{b}, B\right]\right] .
\end{aligned}
$$

The terms quadratic in $B$ in the $\mathcal{H}$ drop out as follows:

$$
\begin{aligned}
& -\frac{1}{4} g_{a b}(i \hbar)^{-1}\left[F^{a}, F^{b}\right] \\
& \quad=-\frac{1}{4} g^{a b} \varepsilon_{a c} \varepsilon_{b d}(i \hbar)^{-3}\left[\left[\Delta_{+}^{c}, B\right],\left[\Delta_{+}^{d}, B\right]\right] \\
& \quad=-\frac{1}{8} g^{a b} \varepsilon_{a c} \varepsilon_{b d}(i \hbar)^{-3}\left(\left[\Delta_{+}^{c},(B, B)_{\Delta_{+}}^{d}\right]+(c \leftrightarrow d)\right)=0 .
\end{aligned}
$$

Here in Eq. (4.16), in the last equality, we have used (4.10) and then Eq. (4.8). The superfield $\Psi$ has the component form

$\Psi(t, \tau, Z)=\exp \left\{\tau_{a}(i \hbar)^{-1} Q^{a}\right\} \Psi_{0}(t, Z)$,

where the zero-component satisfies by itself the Schroedinger equation (4.14) with the Hamiltonian (4.15). The same as in the $\operatorname{Sp}(1)$ case, the arbitrariness in a solution to the quantum master equations

$\Delta_{+}^{a} \Psi_{0}=0, \quad \varepsilon\left(\Psi_{0}\right)=0, \quad \Psi_{0}=\exp \left\{\frac{i}{\hbar} W\right\}$,

is measured by the evolution operator with the Hamiltonian (4.15).

It seems a bit strange that the boson $B$ is restricted so as to satisfy Eq. (4.8), although the standard expression in the right-hand side of the second equality in (4.15) does commute with the $\Delta_{+}^{a}$ as for an arbitrary $B$. The reason is just the second equality (4.15) by itself. In order to clarify the matter, let us consider the definition of the Hamiltonian $\mathcal{H}$ in a natural basis,

$g^{a b}=g_{a b}=:\left(\begin{array}{ll}0 & 1 \\ 1 & 0\end{array}\right), \varepsilon^{a b}=-\varepsilon_{a b}=:\left(\begin{array}{ll}0 & 1 \\ -1 & 0\end{array}\right)$,

so that

$$
\begin{gathered}
D^{1}=\frac{\partial}{\partial \tau_{1}}+\tau_{2} \frac{\partial}{\partial t}, \quad D^{2}=\frac{\partial}{\partial \tau_{2}}+\tau_{1} \frac{\partial}{\partial t}, \\
g^{a b} \varepsilon_{b c}=\left(\begin{array}{cc}
1 & 0 \\
0 & -1
\end{array}\right) .
\end{gathered}
$$

First of all, we have, for the Hamiltonian $\mathcal{H}$, the first equation in (4.15),

$\mathcal{H}=-\frac{1}{2}(i \hbar)^{-1}\left[Q^{1}, Q^{2}\right]$,

where

$Q^{1}=\Delta_{+}^{1}-F^{1}, \quad Q^{2}=\Delta_{+}^{2}-F^{2}$,

$F^{1}=(i \hbar)^{-1}\left[\Delta_{+}^{1}, B\right], \quad F^{2}=-(i \hbar)^{-1}\left[\Delta_{+}^{2}, B\right]$,

so that

$$
\begin{aligned}
\mathcal{H}= & -\frac{1}{2}(i \hbar)^{-2}\left(\left[\Delta_{+}^{1},\left[\Delta_{+}^{2}, B\right]\right]-(1 \leftrightarrow 2)\right. \\
& \left.-(i \hbar)^{-1}\left[\left[\Delta_{+}^{1}, B\right],\left[\Delta_{+}^{2}, B\right]\right]\right) .
\end{aligned}
$$

In order to provide for the operators $Q^{1}$ and $Q^{2},(4.22)$, to commute with the Hamiltonian $\mathcal{H},(4.21)$, both charges (4.22) should be nilpotent,

$\left[\left[\Delta_{+}^{1}, B\right],\left[\Delta_{+}^{1}, B\right]\right]=0, \quad\left[\left[\Delta_{+}^{2}, B\right],\left[\Delta_{+}^{2}, B\right]\right]=0$.

The first and the second equations in (4.25) are exactly Eq. (4.8) at $a=b=1$ and at $a=b=2$, respectively. Now, in the first line in the right-hand side in (4.24) we recognize exactly the standard expression in the right-hand side in the second equality in (4.15). In turn, Eq. (4.8) at $a=1, b=2$, or vice versa, cancels the expression in the second line in (4.24). Thus, we have explained in detail how Eq. (4.8) for the boson operator $B$ do come from the general structure (4.21) of the Hamiltonian $\mathcal{H}$ as constructed for the two nilpotent supercharges $Q^{1}$ and $Q^{2}$. In contrast to the $\operatorname{Sp}(1)$ case, in 
the $S p(2)$ symmetric superfield formalism, Eq. (4.8) are just the price of the higher supersymmetry.

Finally, consider, in the $S p(2)$ case, the composition law similar to the one of (3.11) and (3.12), as for the Hamiltonian (4.15) rewritten as

$\mathcal{H}_{F^{2}}=(i \hbar)^{-1}\left[\Delta_{+}^{1}, F^{2}\right]$,

where $F^{2}$ is given by the second in (4.23). Then the composition law has just the form (3.11), (3.12), with the $\Delta_{+}^{1}$ and the $F^{2}$ standing for the $\Delta$ and the $F$, respectively. Vice versa, we could make use of the $\Delta_{+}^{2}$ and the $F^{1}$ as to stand for the $\Delta$ and the $F$, respectively, when having the Hamiltonian (4.15) rewritten equivalently as

$\mathcal{H}_{F^{1}}=(i \hbar)^{-1}\left[\Delta_{+}^{2}, F^{1}\right]$,

where $F^{1}$ is given by the first in (4.23).

\section{General nilpotency}

Here, we present in both the $S p(1)$ and the $S p(2)$ cases, in parallel, the simplest class of solutions for the Hamiltonian. In the $S p(1)$ case, we strengthen Eq. (2.13) to the nilpotency condition for the fermion $F$,

$\mathcal{H}_{S}=0, \quad G=0, \Rightarrow[F, F]=0$.

Then we have for the Hamiltonian

$\mathcal{H}=(i \hbar)^{-1}[\Delta, F]$.

In the case of $F$ being a function of $Z^{A}$ only, the condition (5.1) is satisfied automatically. In the $S p(2)$ case, we strengthen Eq. (4.12) to the "nilpotency" condition for the boson $B$,

$X^{a}=0, \quad Y=0, \Rightarrow(B, B)_{\Delta_{+}}^{a}=0$.

Then we have for the Hamiltonian

$\mathcal{H}=\frac{1}{2}(i \hbar)^{-2}\left[\Delta_{+}^{a}, \varepsilon_{a b}\left[\Delta_{+}^{b}, B\right]\right]$.

In the case of the $B$ being a function of the fields only, Eq. (5.3) is satisfied automatically.

\section{Heisenberg equations of motion in terms of quantum antibrackets}

Here, we present in both the $S p(1)$ and the $S p(2)$ cases, in parallel, the Heisenberg equations of motion in terms of the quantum antibrackets. Denote by $\Gamma$ the full set of Schroedinger canonical variable operators,

$\Gamma=:\left(Z^{A} ; P_{A}\right)$, and let $\tilde{\Gamma}(t, \tau)$ be the respective superfield Heisenberg canonical variable operators.

In the $S p(1)$ case, the superfield Heisenberg equations of motion have the form

$i \hbar D \tilde{\Gamma}=[\tilde{Q}, \tilde{\Gamma}], \quad i \hbar D \tilde{Q}=[\tilde{Q}, \tilde{Q}]$.

It follows from these equations that [11]

$(i \hbar)^{2} \frac{\partial}{\partial t} \tilde{\Gamma}=-\frac{1}{2}[\tilde{\Gamma},[\tilde{Q}, \tilde{Q}]]=-\frac{2}{3}(\tilde{\Gamma}, \tilde{Q})_{\tilde{Q}}$,

where the quantum 2-antibracket, $(A, B)_{Q}$, is defined by Eq. (3.13), with $Q$, the third in Eq. (2.1), standing for the $\Delta$.

In the $S p(2)$ case, the respective superfield Heisenberg equations of motion have the form

$i \hbar D^{a} \tilde{\Gamma}=\left[\tilde{Q}^{a}, \tilde{\Gamma}\right], \quad i \hbar D^{a} \tilde{Q}^{b}=\left[\tilde{Q}^{a}, \tilde{Q}^{b}\right]$.

It follows from these equations that

$(i \hbar)^{2} \frac{\partial}{\partial t} \tilde{\Gamma}=-\frac{1}{4} g_{a b}\left[\tilde{\Gamma},\left[\tilde{Q}^{b}, \tilde{Q}^{a}\right]\right]=-\frac{1}{3} g_{a b}\left(\tilde{\Gamma}, \tilde{Q}^{b}\right)_{\tilde{Q}}^{a}$,

where the $S p(2)$ vector-valued quantum 2-antibracket, $(A, B)_{Q}^{a}$, is defined by Eq. (4.9), with $Q^{a}$, the first in Eq. (4.3), standing for the $\Delta_{ \pm}^{a}$.

\section{Conclusion}

In the present paper, within the superfield approach, we have proposed the new quantum generating equation (2.1) for the general field-antifield formalism. The three basic fermion objects, the super-time covariant derivative $D$, the odd Laplacian $\Delta$, and the hyper-gauge fermion $F$, enter that linear homogeneous generating equation, in a quite symmetric way. Then, from the generating equation, we have derived the Schroedinger equation (2.7) with the Hamiltonian $\mathcal{H}$, (2.8), commuting with the supercharge $\mathrm{Q}$, the third in (2.1). It follows from the latter property (2.10) that the Hamiltonian $\mathcal{H}$ commutes with the $\Delta$, provided the $\mathcal{H}$ commutes with the $F$, as well. Thus, we have determined the general structure (2.15) of the Hamiltonian (2.8). As usual, the Hamiltonian consists of a singlet component and a $\Delta$-exact component. We have shown that the $\Delta$-exact components (3.1) serve as generators to the quantum master transformations. In turn, we have shown that these generators (3.2) do satisfy the nice composition law (3.11) given by (3.12) in terms of the quantum antibrackets (3.13). We have also presented an $S p(2)$ symmetric extension to the main construction, with specific features caused by the principal fact that all basic equations become $S p(2)$ vector-valued ones. 
Acknowledgements The authors would like to thank Klaus Bering of Masaryk University for interesting discussions. The work of I. A. Batalin is supported in part by the RFBR Grants 17-01-00429 and 1702-00317. The work of P. M. Lavrov is supported in part by the RFBR Grant 16-52-12012-NNIO.

Open Access This article is distributed under the terms of the Creative Commons Attribution 4.0 International License (http://creativecomm ons.org/licenses/by/4.0/), which permits unrestricted use, distribution, and reproduction in any medium, provided you give appropriate credit to the original author(s) and the source, provide a link to the Creative Commons license, and indicate if changes were made.

Funded by SCOAP ${ }^{3}$.

\section{References}

1. I.A. Batalin, G.A. Vilkovisky, Gauge algebra and quantization. Phys. Lett. B 102, 27-31 (1981)

2. I.A. Batalin, G.A. Vilkovisky, Quantization of gauge theories with linearly dependent generators. Phys. Rev. D 28, 2567-2582 (1983)

3. J.A. Schouten, Über differentialcomitanten zweier Kontravarianter Grössen. Proc. Nederl. Acad. Wetensh. Ser. A 43, 449-452 (1940)

4. C. Buttin, Les de'rivations des champs de tenseurs et l'invariant diffe'rentiel de Schouten. C. R. Acad. Sci. Paris. Ser. A B 269, 87 (1969)

5. I.A. Batalin, E.S. Fradkin, Operatorial quantizaion of dynamical systems subject to constraints. A further study of the construction. Ann. Inst. H. Poincare Phys. Theor. 49, 145-214 (1988)

6. G.V. Grigorian, R.P. Grigorian, I.V. Tyutin, Equivalence of Lagrangian and Hamiltonian BRST quantizations: systems with first class constraints. Sov. J. Nucl. Phys. 53, 1058-1061 (1991)

7. I.A. Batalin, K. Bering, P.H. Damgaard, Superfield quantization. Nucl. Phys. B 515, 455-487 (1998)

8. I.A. Batalin, K. Bering, P.H. Damgaard, Superfield formulation of the phase path integral. Phys. Lett. B 446, 175-178 (1999)

9. I.A. Batalin, P.M. Lavrov, Superfield Hamiltonian quantization in terms of quantum antibrackets. Int. J. Mod. Phys. A 31, 16500541-14 (2016)

10. I. Batalin, R. Marnelius, Quantum antibrackets. Phys. Lett. B 434 312-320 (1998)
11. I. Batalin, R. Marnelius, General quantum antibrackets. Theor. Math. Phys. 120, 1115-1132 (1999)

12. I.A. Batalin, P.M. Lavrov, I.V. Tyutin, Finite anticanonical transformations in field-antifield formalism. Eur. Phys. J. C 75, 270-1270-16 (2015)

13. P.M. Lavrov, I.V. Tyutin, Gauge theories of general form. Sov. Phys. J. 25, 639-641 (1982)

14. I.A. Batalin, G.A. Vilkovisky, Closure of the Gauge algebra, generalized Lie equations and Feynman rules. Nucl. Phys. B 234, 106124 (1984)

15. I.A. Batalin, K. Bering, Gauge independence in a higher-order Lagrangian formalism via change of variables in the path integral. Phys. Lett. B 742, 23-28 (2015)

16. I.A. Batalin, P.M. Lavrov, Closed description of arbitrariness in resolving quantum master equation. Phys. Lett. B 758, 54-58 (2016)

17. I.A. Batalin, P.M. Lavrov, General quantum-mechanical setting for field-antifield formalism as a hyper-gauge theory. Mod. Phys. Lett. A 30, 1650167-1-1650167-15 (2016)

18. Y. Kosmann-Schwarzbach, Derived brackets. Lett. Math. Phys. 69, 61-87 (2004)

19. K. Bering, Non-commutative Batalin-Vilkovisky algebras, strongly homotopy Lie algebras, and the Courant bracket. Commun. Math. Phys. 274, 297-341 (2007)

20. I.A. Batalin, P.M. Lavrov, I.V. Tyutin, Covariant quantization of gauge theories in the framework of extended BRST symmetry. J. Math. Phys. 31, 1487-1493 (1990)

21. I.A. Batalin, P.M. Lavrov, I.V. Tyutin, An Sp(2) covariant quantization of gauge theories with linearly dependent generators. J. Math. Phys. 32, 532-539 (1991)

22. I.A. Batalin, P.M. Lavrov, I.V. Tyutin, Remarks on the $S p(2)$ covariant Lagrangian quantization of gauge theories. J. Math. Phys. 32, 2513-2521 (1991)

23. I.A. Batalin, K. Bering, P.M. Lavrov, A systematic study of finite BRST-BV transformations within $W-X$ formulation of the standard and the $S p$ (2)-extended field-antifield formalism. Eur. Phys. J. C 76, 101-1-101-8 (2016) 